\title{
Sex hormone binding globulin concentration as a prepubertal marker for hyperinsulinaemia in obesity
}

\author{
P J Galloway, M D C Donaldson, A M Wallace
}

\begin{abstract}
Background-Overweight children become obese adults who are prone to develop the "metabolic syndrome" and premature coronary arterial disease (CAD).

Aims-To assess whether sex hormone binding globulin (SHBG) is a potential marker for hyperinsulinaemia/insulin resistance in prepubertal obese children.

Methods-Twenty five obese children (body mass index (BMI) >2SD) who warranted investigation on clinical grounds were enrolled. Their insulin response to an oral glucose tolerance test was assessed.

Results-Fourteen children were hyperinsulinaemic. Despite being matched for age and BMI, SHBG concentrations were below the sex related reference range in the hyperinsulinaemic group.

Conclusion-Our results indicate that a subnormal SHBG concentration in a prepubertal child is strongly predictive of hyperinsulinaemia. By measuring the circulating SHBG concentration, it might be possible to identify those at most risk of premature CAD, targeting them for lifestyle changes.

(Arch Dis Child 2001;85:489-491)
\end{abstract}

Keywords: SHBG; insulin; prepubertal; obesity

The current trend of increasing childhood obesity in the industrialised world continues unabated. ${ }^{1-3}$ Childhood obesity carries with it the burden of significant health consequences in adult life, as illustrated by a Finnish study showing that the risk of suffering from the metabolic syndrome (hypertension, hyperinsulinaemia, hyperlipidaemia, and visceral obesity) in adulthood was increased 56-fold (CI: 13 to 504) if obesity began during childhood $^{4}$ (obesity defined as top tertile). Furthermore it has been shown that there is an increased incidence of obesity in children whose parents had early coronary artery disease (CAD). ${ }^{5}$

Obese children most at risk are likely to be those who have inherited the polygenic propensity for hyperinsulinaemia/insulin resistance considered by many to be the central aetiological factor for the metabolic syndrome. ${ }^{6}$ The ability to target such children, and their families, for exercise and nutritional education could reduce their risk of developing the metabolic syndrome and premature CAD. To date, biochemical assessment has relied on prolonged dynamic testing using either the insulin response to a glucose load or by euglycaemic clamp, procedures that are not appropriate for screening children in an obesity clinic.

In addition to androgens and thyroxine, insulin is known to regulate sex hormone binding globulin (SHBG) production by the liver. ${ }^{7}$ Low circulating SHBG concentrations are known to occur in adults as a consequence of hyperinsulinaemia/insulin resistance. ${ }^{8}$ We wished to investigate whether this might also be the case in prepubertal children such that it could provide the basis of a screening test for childhood hyperinsulinaemia.

\section{Patients and methods}

We enrolled 25 prepubertal obese children for oral glucose tolerance testing (OGTT). We defined obese children as those whose body mass index (BMI) was greater than $2 \mathrm{SD}$ from the mean. ${ }^{9}$ All received $45 \mathrm{~g} / \mathrm{m}^{2}$ oral glucose at $9 \mathrm{am}$, after fasting from $10 \mathrm{pm}$ the preceding night. Venous blood samples were collected before the glucose load and at 30 minute intervals for two hours. Plasma was rapidly separated and stored at $-20^{\circ} \mathrm{C}$; insulin was measured by an in house radioimmunoassay with a coefficient of variation of $10 \%$ at 15 $\mathrm{pmol} / \mathrm{l}$. Using the trapezoid rule, the area under the insulin/time curve (AUC) was calculated. We considered values $>100 \mathrm{pmol} / \mathrm{l} / 2 \mathrm{~h}$ diagnostic of insulin resistance as derived locally from adult euglycaemic clamp studies. ${ }^{10}$

We recruited 57 healthy schoolchildren (27 boys, 30 girls; aged 5-10 years), with no evidence of pubertal development, and 71 older healthy children ( 38 boys, 33 girls; aged 10-17 years), for whom pubertal status was not recorded, as a reference paediatric population. At least four samples were obtained from children of each gender for each year of age. All had a venous blood sample collected and analysed for SHBG by immunoradiometric assay (Orion Diagnostica kit supplied by Pharmacia Biosystems Ltd, Milton Keynes) with an interassay coefficient of variation of $7 \%$.

The Yorkhill ethical committee approved this study. Informed and written consent was obtained from the parents of all children who underwent detailed clinical and biochemical investigation.

\section{STATISTICAL ANALYSIS}

Comparison between the hyperinsulinaemic and non-hyperinsulinaemic children involved unpaired $t$ tests on the age and BMI SD scores, 
Table 1 Characteristics of the 25 obese children studied

\begin{tabular}{llll}
\hline & $\begin{array}{l}\text { Exaggerated insulin response } \\
(\text { AUC }>100 \text { pmol/l/2 } h)\end{array}$ & $\begin{array}{l}\text { Normal insulin response } \\
(\text { AUC }<100 \text { pmol/l/2 } h)\end{array}$ & Statistics \\
\hline No. & 14 & 11 & - \\
Age (y) & & $7.6 \pm 2.5$ & $\mathrm{NS}$ \\
$\quad$ Mean \pm SD & $8.3 \pm 2.2$ & $3.2-13$ & \\
$\quad$ Range & $3.5-11.8$ & $5.4,7.5,8.7$ & - \\
$\quad$ Interquartiles & $6.9,8.1,10.4$ & $6: 5$ & $\mathrm{NS}$ \\
Sex (male:female) & $4: 10$ & $3.76 \pm 2.23$ & $\mathrm{p}<0.0025$ \\
BMI SDS (mean \pm SD) & $3.75 \pm 1.63$ & 46 & \\
SHBG (nmol/l) & & $19-75$ & \\
$\quad$ Median & 22 & $41,46,61$ & \\
$\quad$ Range & $9-50$ & & \\
\hline
\end{tabular}

and the Mann-Whitney U test on SHBG concentrations. Data were analysed with Minitab statistical software (Minitab data analysis software, release 8.1, standard version; Minitab Inc., 3081 Enterprise Drive, State College, PA 16801, USA).

Results

In the normal prepubertal healthy schoolchildren group, circulating SHBG ranged from 35 to $168 \mathrm{nmol} / 1$ in boys and 44 to $143 \mathrm{nmol} / 1$ in girls (mean $\pm 2 \mathrm{SD}$ ). These values decreased with increasing age, approaching adult concentrations (6-45 nmol/1 in boys and 30-120 $\mathrm{nmol} / \mathrm{l}$ in girls) by 17 years.

The 25 children who underwent oral glucose tolerance tests had no physical signs of sexual development before testing or on subsequent review four months later. Table 1 shows age, BMI, and SHBG ranges. While the mean age was 7.9 years, the oldest prepubertal child was aged 13 years. Fourteen ( 10 girls, four boys) of the 25 obese subjects showed an exaggerated

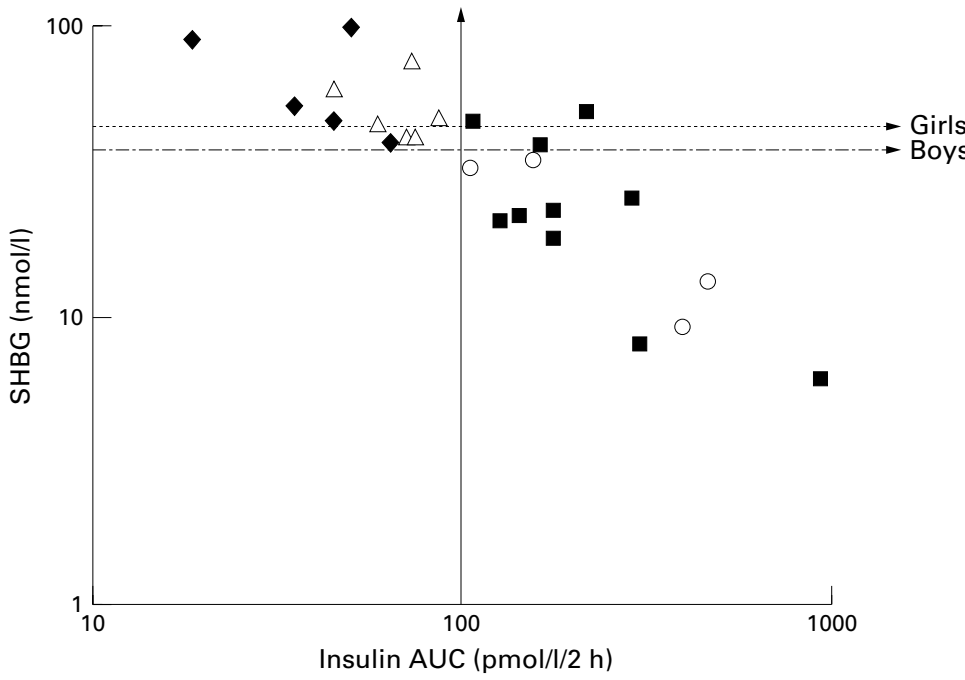

- Non-hyperinsulinaemic females Hyperinsulinaemic females $\triangle$ Non-hyperinsulinaemic males oHyperinsulinaemic males

Vertical line separating insulin response at AUC $100 \mathrm{pmol} / \mathrm{l} / 2 \mathrm{~h}$

…-..- Lower limit of prepubertal female reference range $(44 \mathrm{nmol} / \mathrm{l})$

$\rightarrow$ Lower limit of prepubertal male reference range (35 nmol/l)

Figure 1 Insulin response to a glucose load $\left(45 \mathrm{~g} / \mathrm{m}^{2}\right)$ relative to $S H B C$ concentration. insulin response to oral glucose load (AUC $>100 \mathrm{pmol} / 1 / 2 \mathrm{~h}$ ) consistent with hyperinsulinism. There was no significant difference in age or BMI between the hyperinsulinaemic and non-hyperinsulinaemic groups. Circulating SHBG concentrations were subnormal in all but two patients in the hyperinsulinaemic group (fig 1). Both patients were girls, one of whom had borderline hyperinsulinaemia (AUC $108 \mathrm{pmol} / 1 / 2 \mathrm{~h}$ ). By contrast all but two of the non-hyperinsulinaemic group showed normal SHBG concentrations. Both exceptions were girls with SHBG values of 19 and 36 $\mathrm{nmol} / 1$.

Log transformation showed serum SHBG concentrations were negatively correlated with OGTT insulin AUC $(r=-0.65)$. In our group of obese prepubertal children $(\mathrm{n}=25)$, a circulating SHBG concentration below reference range was $82 \%$ specific in predicting an exaggerated OGTT insulin response with $86 \%$ sensitivity.

\section{Discussion}

Our data support the hypothesis that, as has been shown in adults, ${ }^{8}$ the circulating SHBG concentration in childhood and adolescence is a marker for hyperinsulinaemia/insulin resistance. Circulating SHBG concentrations in our normal population group showed a decrease as puberty approaches, which was more notable in boys. This decrease coincides with the well documented phase of insulin resistance associated with puberty. ${ }^{11}$

We have shown a strong association between low SHBG concentrations with documented hyperinsulinaemia in obese children. None of the children showed evidence of early or precocious puberty during or shortly after the study, and the insulin resistance appeared to be a consequence of being overweight.

Although obesity is clearly a cause of insulin resistance, other factors must be responsible as only $56 \%$ of our obese study group was hyperinsulinaemic. Moreover, the actual degree of obesity in hyperinsulinaemic and nonhyperinsulinaemic children was similar.

Our finding that the circulating SHBG concentration is a better positive predictor of insulin resistance than BMI may facilitate identification of at risk prepubertal children who could be targeted for weight management and lifestyle changes in the hope of reducing their metabolic consequences in later life.

We are extremely grateful to Drs MRH Taylor and CV Holland, Department of Paediatrics, Trinity College, Dublin, and Dr PM Crofton, Royal Hospital for Sick Children, Edinburgh, for assistance in obtaining samples from normal children.

1 Freedman DS, Srinivan SR, Valdez R, et al. Secular increases in relative weight and adiposity among children over two decades: the Bogalusa Heart Study. Pediatrics 1997;319:420-6.

2 Troiano RP, Flegal KM, Kuzmarski RJ, et al. Overweight prevalence and trends for children and adolescents: the National Health and Nutrition Examination Surveys, 1963 to 1991. Arch Pediatr Adolesc Med 1995;149:1085-91.

3 Livingstone MBE. Epidemiology of childhood obesity in Livingstone MBE. Epidemiology of childhood

Europe. Eur f Paediatr 2000;159(suppl 1):514-34.
4 Vanhala M, Vanhala P, Kumpusalo E, et al. Relationship

4 Vanhala $M$, Vanhala $P$, Kumpusalo E, et al. Relationship between obesity from childhood to adulthood and the
metabolic syndrome: population based study. BMF 1998; metabolic
317:319. 
5 Bao W, Srinivasan S, Valdez R, et al. Longitudinal changes in cardiovascular risk from childhood to young adulthood in offspring of parents with coronary artery disease: the Bogalusa Heart Study. $7 A M A 1997 ; 278: 1749-54$.

6 Zimmet P, Boyko EJ, Collier GR, DeCourten M. Etiology of the metabolic syndrome: potential role of insulin resistance, leptin resistance, and other players. Ann N Y Acad Sci 1999;892:25-44.

7 Plymate SR, Matej LA, Jones RE, et al. Inhibition of sex hormone-binding globulin production in human hepatoma (HepG2) cell line by insulin and production. F Clin Endocrinol Metab 1998;67:460-4.
8 Nestler JE. Editorial. Sex hormone-binding globulin: marker for hyperinsulinaemia and/or insulin resistance? $\mathcal{F}$ Clin Endocrinol Metab 1993;76:273-4.

9 Preece MA, Freeman JV, Cole TJ. Sex differences in infancy. $B M F$ 1996;313:1486.

10 Dorrian CA. Human proinsulin and insulin: antibody production, assay development and clinical application. PhD thesis. Faculty of Medicine, University of Glasgow, 1989

11 Potau N, Ibanez L, Rique S, Carroscosa A. Pubertal changes in insulin secretion and peripheral insulin sensitivity. Horm Res 1997;48:219-26.

\section{The trauma of acne}

That acne in adolescence may cause emotional trauma seems almost self-evident but the few studies reported on the subject can be criticised on grounds of patient selection or age-inappropriate psychological assessments. A study in Nottingham (A Smithard and colleagues. British fournal of Dermatology 2001;145:274-9) has attempted to correct these deficiencies.

A total of 317 pupils aged $14-16$ attending a single comprehensive school completed an age-appropriate questionnaire aimed at measuring emotional health (the Strengths and Difficulties Questionnaire (SDQ)) and another questionnaire about knowledge of acne and use of treatments. They were then examined using a standardised technique (the Leeds Acne Grading Technique). Half of the pupils (158/317) had acne, 70 very mild, 54 mild, and 34 moderate or severe. More severe acne was associated with greater emotional stress (higher SDQ score) in boys but not in girls although in general girls had higher SDQ scores irrespective of the presence or severity of acne. Overall, young people with mild or worse acne were almost twice as likely as those with no or very mild acne to have a borderline or abnormal SDQ score.

These young people had little knowledge of the causes of acne and their knowledge did not vary according to the presence or severity of acne. They were more likely to discuss the problem with parents or friends than to consult a doctor. Of those with mild or worse acne, only a third had consulted a doctor and another third denied they had acne. Since acne is a treatable condition, education in adolescence might improve treatment uptake and also improve the mental health of acne sufferers.

ARCHIVIST 